\title{
Longitudinal Discriminant Analysis of Hemoglobin Level for Predicting Preeclampsia
}

\author{
Malihe Nasiri ${ }^{1}$; Soghrat Faghihzadeh ${ }^{2, *}$; Hamid Alavi Majd ${ }^{3}$; Farid Zayeri ${ }^{3}$; Noorosadat \\ Kariman $^{4}$; Nastaran Safavi Ardebili ${ }^{5}$ \\ ${ }^{1}$ Department of Biostatistics, Faculty of Medical Sciences, Tarbiat Modares University, Tehran, IR Iran \\ ${ }_{3}^{2}$ Department of Biostatistics, Faculty of Medical Sciences, Zanjan University of Medical Sciences, Zanjan, IR Iran \\ ${ }^{3}$ Department of Biostatistics, Faculty of Paramedical, Shahid Beheshti University of Medical Sciences, Tehran, IR Iran \\ ${ }_{5}^{4}$ Department of Midwifery, Faculty of Nursing and Midwifery, Shahid Beheshti University of Medical Sciences, Tehran, IR Iran \\ 5 Department of Midwifery, Faculty of Nursing and Midwifery, Shahid Beheshti University of Medic \\ ${ }^{*}$ Corresponding Author: Soghrat Faghihzadeh, Department of Biostatistics, Faculty of Medical Sciences, Zanjan University of Medical Sciences, Zanjan, IR Iran. Tel: +98-2414240301, \\ E-mail: s.faghihzadeh@zums.ac.ir
}

Received: April 15, 2014; Revised: May 10, 2014; Accepted: June 9, 2014

Background: Preeclampsia is one of the most serious complications during pregnancy with important effects on health of mother and fetus that causes maternal and fetal morbidity and mortality. This study was performed to evaluate whether high levels of hemoglobin may increase the risk of preeclampsia.

Objectives: The present study aimed to predict preeclampsia by the hemoglobin profiles through longitudinal discriminant analysis and comparing the error rate of discrimination in longitudinal and cross sectional data.

Patients and Methods: In a prospective cohort study from October 2010 to July 2011, 650 pregnant women referred to the prenatal clinic of Milad Hospital in Tehran were evaluated in 3 stages. The hemoglobin level of each woman was measured in the first, second, and third trimester of pregnancy by an expert technician. The subjects were followed up to delivery and preeclampsia was the main outcome under study. The covariance pattern and linear-mixed effects models are common methods that were applied for discriminant analysis of longitudinal data. Also Student t, Mann-Whitney U, and chi-square tests were used for comparing the demographic and clinical characteristics between two groups. Statistical analyses were performed using the SAS software version 9.1.

Results: The prevalence rate of preeclampsia was $7.2 \%$ (47 women). The women with preeclampsia had a higher mean of hemoglobin values and the difference was $0.46 \mathrm{~g} / \mathrm{dL}(\mathrm{P}=0.003)$. Also the mean of hemoglobin in the first trimester was higher than that of the second trimester, and was lower than that of the third trimester and the differences were significant $(\mathrm{P}=0.015$ and $\mathrm{P}<0.001$, respectively). The sensitivity for longitudinal data and cross-sectional data in three trimesters was $90 \%, 67 \%, 72 \%$, and $54 \%$ and the specificity was $88 \%, 55 \%, 63 \%$, and $50 \%$, respectively.

Conclusions: The levels of hemoglobin can be used to predict preeclampsia and monitoring the pregnant women and its regular measure in 3 trimesters help us to identify women at risk for preeclampsia.

Keywords: Discriminant Analysis; Preeclampsia; Hemoglobin

\section{Background}

Preeclampsia is a medical condition in which hypertension arises in pregnancy (pregnancy-induced hypertension) in association with significant amounts of protein in the urine. Preeclampsia is diagnosed when a pregnant woman develops high blood pressure (two separate readings taken at least 6 hours apart of $140 / 90 \mathrm{mmHg}$ or more) and $300 \mathrm{mg}$ of protein in a 24-hour urine sample (proteinuria) that occurs after 20 weeks of gestation (1, 2). Preeclampsia is one of the most serious complications during pregnancy with important effects on the health of mother and fetus that causes maternal and fetal morbidity and mortality $(3,4)$. After infection and bleeding, preeclampsia is the third cause of death in pregnant women $(5,6)$. Furthermore, it has significant undesirable effects on fetus, including decreased amniotic fluid levels, de-

creased fetal growth, placental abruption, and intrauterine fetal demise (7).

Although 3\% to $10 \%$ of pregnancies are affected with preeclampsia, it is estimated to be $20 \%$ in developing countries $(8,9)$. A study in Tehran showed a prevalence rate of $6.4 \%$ for preeclampsia in 2010 (6). First pregnancy, mother's age (younger than 18 and older than 35), previous and family history, chronic hypertension, diabetes, renal disease, multiple gestations, and obesity are some of the reported risk factors for preeclampsia $(2,10)$. The etiology of preeclampsia is unknown but may be associated with immune system dysfunction, genetic factors, placental abnormalities, and low antitoxic protective mechanisms because of low protein (albumin) levels in the blood (1113). Delivery of the fetus is the only known cure for pre-

Copyright ( 2 2015, Iranian Red Crescent Medical Journal. This is an open-access article distributed under the terms of the Creative Commons Attribution-NonCommercial 4.0 International License (http://creativecommons.org/licenses/by-nc/4.0/) which permits copy and redistribute the material just in noncommercial usages, provided the original work is properly cited. 
eclampsia. Therefore, early detection of this problem, which involves careful monitoring of both mother and fetus is an important issue (14).

To detect preeclampsia, a variety of quantities are measured at examinations. Hemoglobin commonly measured during the pregnancy is one of these quantities (1519). According to recent reports, the levels of hemoglobin can help to predict preeclampsia. Levels that are abnormally high may be an important alarm for preeclampsia (19-24). In addition, other researchers showed that high levels of hemoglobin may be associated with the increased risk of diabetes, hypertension in pregnancy, preterm delivery, and low birth weight. Thus, checking the hemoglobin is important during the pregnancy for early diagnosis of these problems $(19,20,25,26)$.

In diagnostic medical research, discriminant analysis is usually applied for evaluating the ability of biomarkers to classify patients into different groups. Commonly, biomarkers are measured at a single time for each patient and traditional discriminant analysis may be a feasible approach in this case for classifying the subjects and predicting the diseases under study (27). Fisher linear discriminant function is the usual method in the classic discriminant analysis, which can be used in cross sectional studies. However in longitudinal studies, there are situations that researchers wish to analyze longitudinal biomarkers for predicting the outcome in discriminant analysis.

In these areas, repeated measures of the biomarkers are used for early diagnosis of a specific disease. The covariance pattern and linear mixed-effects models are common methods in discriminant analysis of the longitudinal data (28-30).

\section{Objectives}

The present study aimed to predict preeclampsia by the hemoglobin profiles through longitudinal discriminant analysis and calculating the AIC (Akaike's information criterion), sensitivity, specificity, and misclassification error rate of this approach. We also aimed to compare the predicting ability of covariance pattern and linear mixed-effects models in our data set. Comparing the error rate of discrimination in longitudinal and cross sectional data was another purpose in this study.

\section{Patients and Methods}

In a prospective cohort study from October 2010 to July 2011, 650 pregnant women who were referred to the prenatal clinic of Milad Hospital in Tehran, Iran were evaluated in 3 stages. All women referred to the laboratory and the hemoglobin level of each woman was measured in the first, second, and third trimester of pregnancy by an expert technician. The subjects were followed up to delivery and the presence of preeclampsia was the main outcome under study.

The inclusion criteria were singleton pregnancy, gestational age of 13 weeks or less, maternal age of 18-35 years, parity of 3 or less, lack of systemic diseases, including diabetes, chronic hypertension and cardiovascular disease, chronic renal disease, gastroenterology disease, thyroid, epilepsy, hemoglobinopathies and mental disorders, lack of any history of smoking, alcohol use, non-routine drugs use during the present pregnancy, and lack of any history of preeclampsia in pervious pregnancy. The exclusion criteria were known fetal anomalies, polyhydramnios, oligohydramnios, placenta previa, abruption placenta, abortion, and stillbirth.

Based on the mentioned criteria, 700 subjects were included and 50 subjects were excluded from the study. The study protocol was approved by the Ethics Committee of Shahid Beheshti University of Medical Sciences issued on 31st August 2010 and written informed consent was obtained from the participants after comprehensive explanation of the procedure involved.

\subsection{Statistical Analysis}

As mentioned before, the present study aimed to predict the presence of preeclampsia in pregnant women based on their hemoglobin levels in 3 trimesters. There are a variety of statistical methods for assessing the power of different tests for predicting a binary outcome. For instance, classic discriminant analysis for cross-sectional data, covariance pattern model, and mixed-effects models for longitudinal data are well-known statistical methods in this context (28).

Discriminant analysis includes multivariate techniques for classifying observations into pre-defined groups. In classical discriminant analysis, observations only measure one time and an unstructured pattern is assumed as the covariance matrix, and then the Fisher linear discriminant function can be used for classification and discrimination (28).

Covariance pattern model assumes a functional form such as compound symmetry (CS) or first-order autoregressive ("AR (1)") for variance-covariance and correlation matrix. These structures for the covariance matrix in the covariance pattern model enable discriminant analysis to consider correlation between observations, which should be assumed in the longitudinal data $(30,31)$.

The mixed-effects model supposes correlation structures such as CS or AR (1) for estimating the correlation between repeated measures data and also time is incorporated as a covariate in this model. Furthermore, a random subject effect is assumed in the model for considering the within-subject variance. This variance for one particular subject can be different by each subject and let the model to capture the variability and cause more precise for estimation the parameters. Also mixed-effects model will allow discriminant analysis to cope with missing data (29-32).

The accuracy of the classification is usually described by the misclassification error rate (MER); MER is the error rate that estimates the proportion of study participants assigned to groups incorrectly (28). Also evaluating the 
AIC, sensitivity, and specificity rates are practical criterions for comparing different models and assessing the precision of various methods in longitudinal discriminant analysis. Hence, the best model for classifying the patients into groups is a model with the least MER and AIC as well as the most sensitivity and specificity.

Statistical analyses were performed using the SAS software version 9.1. Kolmogorov-Smirnov test was used to test the normality in continuous variables. Student t, Mann-Whitney U, and chi-square tests were used for comparing the demographic and clinical characteristics between two groups. P values of less than 0.05 were considered statistically significant.

\section{Results}

In general, a total sample of 650 pregnant women was studied. The mean \pm SD age of these women was $27.37 \pm$ 3.91, ranged from 18 to 35 years. Among them, 309 women (47.5\%) were experiencing the first pregnancy and 341 (52.5\%) women were experiencing the second or third pregnancy. About $27.8 \%$ of these women had academic education. Body mass index for 332 women (51.1\%) was at the normal range (19-25). The percentage of women that used food supplement (including folic acid, iron, calcium and vitamin) during pregnancy, were 93.2\%, 95.2\%, 64.3\% and $76.3 \%$ respectively. The prevalence rate of preeclampsia was $7.2 \%$ (47 women).

Tables 1 and 2 compare the characteristics of women with or without preeclampsia. There was no significant difference in the demographic and clinical character- istics between two groups (presence or absence of preeclampsia) $(\mathrm{P}>0.05)$.

The hemoglobin level was used in three stages as the longitudinal response. Figure 1 shows the mean changes of hemoglobin in three trimesters for two groups. It appears that there is a time trend for the levels of hemoglobin and the mean of hemoglobin is decreasing in the second trimester and then increasing in the third trimester. The mean hemoglobin level is lower for the women without preeclampsia than that of the other group at 3 times, so it seems to be a good separation between two groups suggesting discriminant analysis a useful approach for our data.

\begin{tabular}{|c|c|c|}
\hline Continuous Variable & Mean \pm SD & PValue \\
\hline Age, $y$ & & 0.11 \\
\hline With preeclampsia & $28.32 \pm 4.55$ & \\
\hline Without preeclampsia & $27.28 \pm 3.84$ & \\
\hline BMI, $\mathrm{Kg} / \mathrm{m}^{2}$ & & 0.15 \\
\hline With preeclampsia & $25.79 \pm 4.44$ & \\
\hline Without preeclampsia & $24.88 \pm 4.43$ & \\
\hline Diastolic blood pressure, $\mathrm{mmHg}$ & & 0.40 \\
\hline With preeclampsia & $65.5 \pm 9.59$ & \\
\hline Without preeclampsia & $64.18 \pm 7.19$ & \\
\hline Systolic blood pressure, $\mathrm{mmHg}$ & & 0.27 \\
\hline With preeclampsia & $104.62 \pm 11.86$ & \\
\hline Continuous Variable & $102.12 \pm 9.78$ & \\
\hline
\end{tabular}

Table 2. The Clinical Characteristics Between Two Groups a

\begin{tabular}{|c|c|c|c|}
\hline Categorical Variable & With Preeclampsia & Without Preeclampsia & P Value \\
\hline Pregnancy & & & 0.29 \\
\hline First & $23(48.9)$ & $275(45.6)$ & \\
\hline Second & $15(31.9)$ & $251(41.6)$ & \\
\hline Third & $9(19.2)$ & $77(12.8)$ & \\
\hline Education & & & 0.71 \\
\hline Diploma or lower & $35(74.5)$ & $434(71.97)$ & \\
\hline Academic & $12(25.5)$ & $169(28.03)$ & \\
\hline Folic acid & & & 0.86 \\
\hline Yes & $45(95.7)$ & $574(95.2)$ & \\
\hline No & $2(4.3)$ & $29(4.8)$ & \\
\hline Iron & & & 0.88 \\
\hline Yes & $46(97.9)$ & $592(98.2)$ & \\
\hline No & $1(2.1)$ & $11(1.8)$ & \\
\hline Calcium & & & 0.87 \\
\hline Yes & $32(68)$ & $400(66.3)$ & \\
\hline No & $15(32)$ & $203(33.7)$ & \\
\hline Vitamin & & & 0.53 \\
\hline Yes & $35(74.5)$ & $478(79.3)$ & \\
\hline No & $12(25.5)$ & $125(20.7)$ & \\
\hline
\end{tabular}

\footnotetext{
${ }^{\mathrm{a}}$ Data are presented as No. (\%).
} 
The mean $( \pm$ SD) hemoglobin level for two groups of with preeclampsia or without preeclampsia were, 12.88 $( \pm 1.20) \mathrm{g} / \mathrm{dL}$ and $12.63( \pm 1.03) \mathrm{g} / \mathrm{dL}$ in the first period, $12.56( \pm 0.98) \mathrm{g} / \mathrm{dL}$ and $11.93( \pm 1.04) \mathrm{g} / \mathrm{dL}$ in the second period and $12.84( \pm 1.39) \mathrm{g} / \mathrm{dL}$ and $12.52( \pm 1.07) \mathrm{g} / \mathrm{dL}$ in the third period, respectively. In model fitting, the women's hemoglobin was assumed as the response variable and preeclampsia and time as the covariates. The results are shown in Table 3. According to Table 3, the women with preeclampsia had a higher mean of hemoglobin values and the difference was 0.46. Also it appears that there is the time trend; mean of hemoglobin level in trimester 1 was higher and in trimester 2 was lower than that of trimester 3.

As mentioned in the previous section, we fitted the co- variance pattern and linear mixed-effects models with unstructured, AR (1) and CS structures in longitudinal discriminant analysis. The discrimination results are given in Table 4. Akaike's information criterion (AIC), misclassification error rates (MER), sensitivity and specificity for all the models described. Also negative predictive value (NPV), positive predictive value (PPV), positive and negative likelihood ratios (LR) were explained in Table 5.

In the next step, the receiver operating characteristics (ROC) curves for longitudinal data, trimesters 1, 2, and 3 are illustrated in Figure 2 the areas under the ROC curve were $93 \%, 59 \%, 68 \%$, and 54\%, respectively. From our results, the sensitivity values for longitudinal model and three trimesters were $90 \%, 67 \%, 72 \%$, and $54 \%$. Also the specificity values were $88 \%, 55 \%, 63 \%$, and $50 \%$, respectively.

Table 3. Longitudinal Model Results for Predicting Hemoglobin Levels in 3 Trimesters for 2 Groups a

\begin{tabular}{lccc}
\hline Variables & Estimate & SE & PValue \\
\hline Without preeclampsia & -0.46 & 0.128 & 0.0003 \\
With preeclampsia & & Reference category & 0.015 \\
Trimester 1 & 0.109 & 0.048 & $<0.001$ \\
Trimester 2 & -0.557 & 0.046 & \\
Trimester 3 & & Reference category &
\end{tabular}

a Abbreviations: SE, Standard Error.

Table 4. Fit Statistics (AIC), Misclassification Error Rate (MER), Sensitivity and Specificity for the Mixed Effects and Covariance Pattern Model With 3 Correlation Structures

\begin{tabular}{lccccc}
\hline Model & Correlation Structure & AIC & MER $^{\text {a }}$ & Sensitivity $^{{ }^{a}}$ & Specificity $^{\text {a }}$ \\
\hline Covariance pattern & CS & 4000.3 & 34.5 & 71 & 60 \\
Mixed effects & CS & 3997.6 & 11 & 90 & 88 \\
Covariance pattern & AR $(1)$ & 4019.5 & 38 & 67 & 57 \\
Mixed effects & AR $(1)$ & 4017.5 & 36 & 67 & 61 \\
Covariance pattern & Unstructured & 4003.4 & 36.5 & 67 & 60 \\
Mixed effects & Unstructured & 4002.3 & 22 & 85 & 71 \\
\hline
\end{tabular}

a Data are presented as \%.

Table 5. Negative Predictive Value (NPV), Positive Predictive Value (PPV), Positive and Negative Likelihood Ratios (LR) for the Mixed Effects and Covariance Pattern Model With 3 Correlation Structures

\begin{tabular}{lccccc}
\hline Model & Correlation Structure & NPV $^{\mathrm{a}}$ & PPV $^{\mathrm{a}}$ & PLR & NLR $^{-}$ \\
\hline Covariance pattern & CS & 67 & 64 & 1.77 & 0.48 \\
Mixed effects & CS & 90 & 88 & 7.5 & 0.11 \\
Covariance pattern & AR $(1)$ & 63 & 52 & 1.56 & 0.58 \\
Mixed effects & AR $\quad(1)$ & 65 & 63 & 1.72 & 0.54 \\
Covariance pattern & Unstructured & 65 & 63 & 1.67 & 0.55 \\
Mixed effects & Unstructured & 83 & 75 & 2.93 & 0.21 \\
\hline
\end{tabular}

a Data are presented as \%. 
Nasiri M et al.

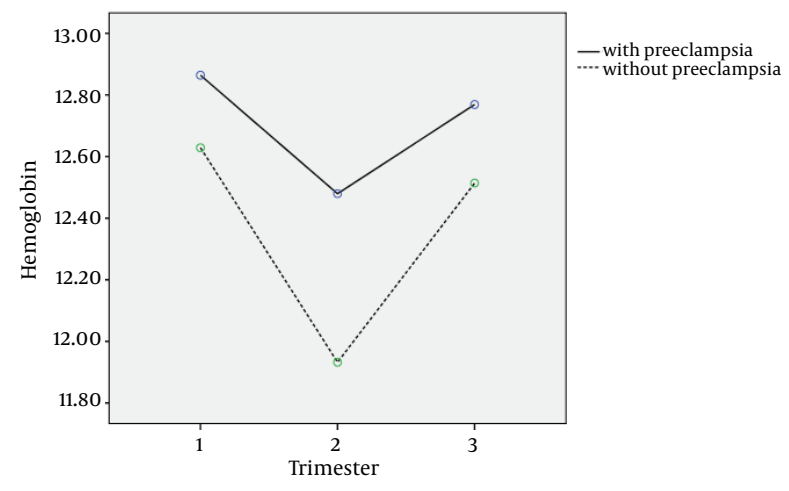

Figure 1. Time Trend of Mean Hemoglobin in Women With and Without Preeclampsia

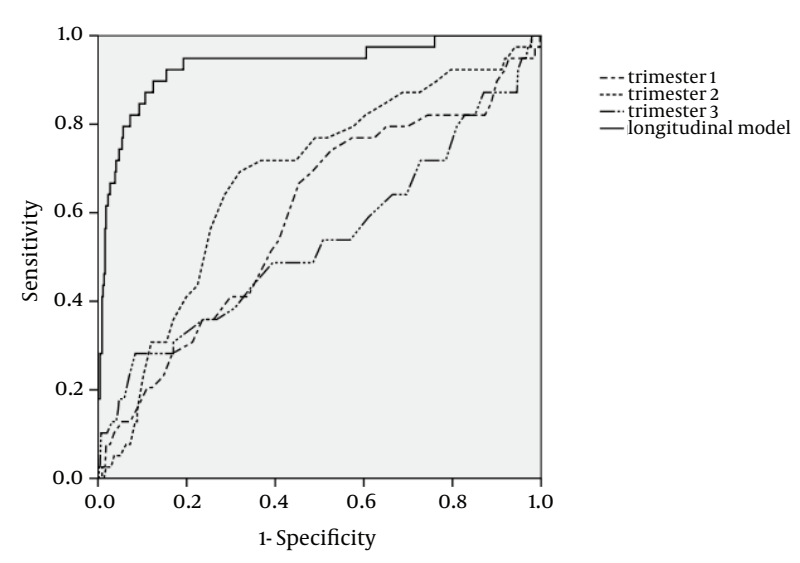

Figure 2. ROC Curves for Longitudinal and Cross Sectional Data in 3 Trimesters

\section{Discussion}

Preeclampsia is the third reason of death in pregnant women and early detection of this disease is important in diagnostic medical research; so this study was performed to predict preeclampsia by the longitudinal hemoglobin data and evaluating the ability of this marker for premature diagnosis of preeclampsia.

Several studies evaluated some risk factors for preeclampsia. Kashanian et al. showed that preexisting preeclampsia $(\mathrm{P}=0.004)$ was a risk factor, whereas parity more than $3(P=0.007)$ and anemia $(P=0.01)$ were protective for preeclampsia (10). Direkvand-Moghadam et al. studied the role of some risk factors as predictors associated with preeclampsia among Iranian women using logistic regression. They concluded the history of preeclampsia, hypertension, and infertility could predict preeclampsia with an increased odds ratio (OR was 5.46, 2.34 and 3.07, respectively)(9). Also Allahyari et al. showed that factors such as fulfillment of university education, marriage more than once, high blood pressure during the 24 to 28 weeks of gestation, being a housekeeper and increase in BMI raised the risk of preeclampsia $(\mathrm{P}<0.05)$ (6). In our study, however, there was no significant difference in age, BMI, systolic and diastolic blood pressure, parity, education, and using food supplement between two groups, (presence or absence of preeclampsia) ( $\mathrm{P}>$ 0.05).

Various quantities are commonly measured during the pregnancy and hemoglobin level is one of them. The high levels of hemoglobin may increase the risk of preeclampsia. A variety of cross sectional studies have performed to investigate the relationship between the levels of hemoglobin and preeclampsia. Phaloprakarn et al. and Chang et al. showed that high hemoglobin in the first and third trimester is associated with preeclampsia $(\mathrm{P}<0.01)(20$, 33). Our results were similar to their findings and showed that the women with preeclampsia had a higher mean of hemoglobin values and the difference was $0.46 \mathrm{~g} / \mathrm{dL}$. Also, Khoigani et al. conducted a study in the first and second half of the pregnancy separately. They concluded hemoglobin levels in the first half of pregnancy can predict preeclampsia ( $\mathrm{P}=0.024)$, but they found no significant association in the second half of the pregnancy ( $P$ $>0.05$ ) (34). In our study, however, the second trimester was more informative than other trimesters. In a study by Alavi, the ability of hemoglobin for predicting the preeclampsia was assessed by calculating the sensitivity and specificity in the first trimester. According to the study of Alavi et al. the cut off point for hemoglobin was 12.45 and the sensitivity and specificity were reported at 0.85 and 0.43 in the first trimester. Shojaeian has also reported the sensitivity and specificity in the second trimester $(5,14)$; the sensitivity and specificity value in their study were 0.72 and 0.57 , respectively. In our study, according to cross sectional data, the sensitivity was 0.67 and 0.72 in the first and second trimester, respectively and the specificity was 0.55 and 0.63 at the corresponding times, respectively. Thus, the specificity was greater in our study than their study in two trimesters, however, in the first trimester, the sensitivity was lower than that of the researches mentioned and in the second trimester, the sensitivity value was similar to them.

At this point, there is a controversial issue; in which trimesters the hemoglobin can better predict preeclampsia. For example in this study, we have a better discrimination in the second trimester compared to first and third ones; because the changes of hemoglobin is higher at this time and the differences in hemoglobin level between two groups is greater in the second trimester. However for some pregnant women, the level of hemoglobin in the first or the third trimester may be significant and this quantity is higher for them compared to the normal group in these trimesters. Furthermore there is a significant time trend for the hemoglobin in three trimesters $(\mathrm{P}<0.05)$.

Thus, combining the trimesters and applying the longitudinal hemoglobin data can increase the accuracy of 
discrimination. Longitudinal discriminant analysis is a useful method to combine three trimesters and expected to acquire a more correct classification and lower error rate. Our findings showed that sensitivity and specificity values for longitudinal model were 0.9 and 0.88 and the area under the ROC curve was $93 \%$. Thus, this study confirmed that in longitudinal data, the error rate of classification is lower and the sensitivity and specificity are greater than that of cross sectional data. Thus the strong point of our study is assuming the time trend in the model and supposing the hemoglobin in three trimesters simultaneously for discriminant analysis and predicting preeclampsia that eventually results in more accuracy. However, in longitudinal discriminant analysis, it is not possible to detect the best cut off point for the hemoglobin level like cross sectional data, because we had the longitudinal hemoglobin data in three trimesters, which makes it a weak point in our research.

In the model fitting, the mixed effects models had a much better predicting ability in comparison to covariance pattern models for all the correlation structures and the mixed-effects model with CS correlation structure on the repeated measures was the best model. This model had the lowest misclassification error rate and AIC, and the largest sensitivity and specificity. In longitudinal discriminant analysis, time trend was assumed by the correlation structures and CS correlation structure was the best structure in all models.

In general, longitudinal discriminant analysis is an efficient method for predicting the outcome in longitudinal data and taking into consideration the time trend in the model provides more precise classification than a cross sectional study. Thus, this method may be practical for time varying markers such as hemoglobin for early detection of diseases. Because of the high sensitivity and specificity, the hemoglobin may be a good marker for early diagnosis of preeclampsia. Therefore, monitoring the pregnant women and measuring regularly their hemoglobin level in 3 trimesters, help us identify the women at risk for preeclampsia.

\section{Acknowledgements}

The authors would like to thank the contribution of Tarbiat Modares University and Delivery Unit of Milad Hospital in this study. Also, we thank the editor, and the reviewers for their constructive comments, which helped to substantially improve this manuscript.

\section{Funding/Support}

Project was fully sponsored by Tarbiat Modares University, Tehran, IR Iran.

\section{References}

1. Khan ZH. Preeclampsia/eclampsia: an insight into the dilemma of treatment by the anesthesiologist. Acta Med Iran. 2011;49(9):564-74.
2. Savaj S, Vaziri N. An overview of recent advances in pathogenesis and diagnosis of preeclampsia. Iran J Kidney Dis. 2012;6(5):334-8.

3. Masoura S, Kalogiannidis IA, Gitas G, Goutsioulis A, Koiou E, Athanasiadis A, et al. Biomarkers in pre-eclampsia: a novel approach to early detection of the disease. J Obstet Gynaecol. 2012;32(7):609-16.

4. Fayyad AM, Harrington KF. Prediction and prevention of preeclampsia and IUGR. Early Hum Dev. 2005;81(11):865-76.

5. Shojaeian Z, Sadeghian HR, Negahban Z. Prediction value of hemoglobin and hematocrit concentration during second trimester in diagnosis of preeclampsia. Iran J obstet gyn infertil. 2010;12(4):13-7.

6. Allahyari E, Foroushani AR, Zeraati H, Mohammad K, Taghizadeh Z. A Predictive Model for the Diagnosis of Preeclampsia. J Reprod Infertil. 2010;10(4):261-7.

7. Gharesi-Fard B, Jafarzadeh L, Zolghadri J, Haghbin H. Soluble CD30 in normal pregnancy, pre-eclampsia and recurrent pregnancy loss. Iran J Immunol. 2012;9(4):234-40.

8. Sobande AA, Eskandar M, Bahar A, Abusham A. Severe pre-eclampsia and eclampsia in Abha, the south west region of Saudi Arabia. J Obstet Gynaecol. 2007;27(2):150-4.

9. Direkvand-Moghadam A, Khosravi A, Sayehmiri K. Predictive factors for preeclampsia in pregnant women: a unvariate and multivariate logistic regression analysis. Acta Biochim Pol. 2012;59(4):673-7.

10. Kashanian M, Baradaran HR, Bahasadri S, Alimohammadi R. Risk factors for pre-eclampsia: a study in Tehran, Iran. Arch Iran Med. 2011;14(6):412-5.

11. Goldenstein H, Levy N, Levy A. Involvement of haptoglobin in prevention of oxidative stress cause by hemoglobin in preeclampsia. Adv Biosci Biotech. 2012;3(7):1037-42.

12. Yaghmaei M, Salimi S, Mokhtari M, Naghavi A, Saravani M, Farajian Mashhadi F. Endothelial nitric oxide synthase gene Glu298Asp polymorphism and risk of preeclampsia in South East of Iran. Afr J Biotech. 2013;10(52):10712-7.

13. Namavar Jahromi B, Rafiee S. Coagulation Factors in Severe Preeclampsia. Iran Red Crescent Med J. 2009;11(3):321-4.

14. Alavi Majd H. The relationship between hemoglobin and hematocrit in the first trimester of pregnancy and preeclampsia. Arak Med Univ J. 2011;14(4):1-9.

15. Vahdat M, Kashanian M, Sariri E, Mehdinia M. Evaluation of the value of calcium to creatinine ratio for predicting of pre-eclampsia. J Matern Fetal Neonatal Med. 2012;25(12):2793-4.

16. Myatt L, Miodovnik M. Prediction of preeclampsia. Semin Perina tol.1999;23(1):45-57.

17. Moretti M, Phillips M, Abouzeid A, Cataneo RN, Greenberg J. Increased breath markers of oxidative stress in normal pregnancy and in preeclampsia. Am J Obstet Gynecol. 2004;190(5):1184-90.

18. Espinoza J. Recent biomarkers for the identification of patients at risk for preeclampsia: the role of uteroplacental ischemia. Expert Opin Med Diagn. 2012;6(2):121-30.

19. Mello G, Parretti E, Cioni R, Lagozio C, Mealli F, Pratesi M. Individual longitudinal patterns in biochemical and hematological markers for the early prediction of pre-eclampsia. J Matern Fetal Neonatal Med. 2002;11(2):93-9.

20. Phaloprakarn C, Tangjitgamol S. Impact of high maternal hemoglobin at first antenatal visit on pregnancy outcomes: a cohort study. J Perinat Med. 2008;36(2):115-9.

21. von Tempelhoff GF, Heilmann L, Rudig L, Pollow K, Hommel G, Koscielny J. Mean maternal second-trimester hemoglobin concentration and outcome of pregnancy: a population-based study. Clin Appl Thromb Hemost. 2008;14(1):19-28.

22. Poulsen HF, Mortensen PE. [Hemoglobin concentration prior to the 20th week of pregnancy correlated with complications in the third trimester]. Ugeskr Laeger. 1990;152(14):1010-1.

23. Harding K, Evans S, Newnham JP. The prediction of pregnancy outcome by haemoglobin measurement before 20 weeks' gestation. J Obstet Gynaecol. 1997;17(1):33-8.

24. Laflamme EM. Maternal hemoglobin concentration and pregnancy outcome: a study of the effects of elevation in el alto, bolivia. Mcgill J Med. 2011;13(1):47.

25. Jasovic-Siveska E, Jasovic V. Prediction of mild and severe pre- 


\section{Nasiri M et al.}

eclampsia with blood pressure measurements in first and second trimester of pregnancy. Ginekol Pol. 2011;82(11):845-50.

26. Amburgey OA, Ing E, Badger GJ, Bernstein IM. Maternal hemoglobin concentration and its association with birth weight in newborns of mothers with preeclampsia.J Matern Fetal Neonatal Med. 2009;22(9):740-4.

27. Kohlmann M, Held L, Grunert VP. Classification of therapy resistance based on longitudinal biomarker profiles. Biom J. 2009;51(4):610-26.

28. Lix LM, Sajobi TT. Discriminant analysis for repeated measures data: a review. Front Psychol. 2010;1:146.

29. Wernecke KD, Kalb G, Schink T, Wegner B. A Mixed Model Approach to Discriminant Analysis with Longitudinal Data. Biometric J. 2004;46(2):246-54.

30. Roy A, Khattree R. Discrimination and Classification with Re- peated Measures Data under Different Covariance Structures. Commun Stat Simul Comp. 2005;34(1):167-78.

31. Marshall G, De la Cruz-Mesia R, Quintana FA, Baron AE. Discriminant analysis for longitudinal data with multiple continuous responses and possibly missing data. Biometrics. 2009; 65(1):69-80.

32. Tomasko L, Helms RW, Snapinn SM. A discriminant analysis extension to mixed models. Stat Med.1999;18(10):1249-60.

33. Chang SC, O'Brien KO, Nathanson MS, Mancini J, Witter FR. Hemoglobin concentrations influence birth outcomes in pregnant African-American adolescents. J Nutr. 2003;133(7):2348-55.

34. Khoigani MG, Goli S, Hasanzadeh A. The relationship of hemoglobin and hematocrit in the first and second half of pregnancy with pregnancy outcome. Iran J Nurs Midwifery Res. 2012;17(2 Suppl 1):S165-70. 